\title{
Solution for a Space-time Fractional Diffusion Equation
}

\author{
Qiyu Liu ${ }^{1,}$ and Longjin $\mathrm{Lv}^{2, *}$ \\ ${ }^{1}$ College of Information Science and Technology, Beijing University of Chemical Technology, Beijing 100029, China \\ ${ }^{2}$ Ningbo Institute of Technology, Zhejiang University, Ningbo 315100, China \\ ${ }^{*}$ Corresponding author
}

\begin{abstract}
This work focuses on investigating the solutions for a generalized fractional diffusion equation. This equation presents space and time fractional derivatives, includes an absorbent term and a linear external force, takes a time-dependent diffusion coefficient into account, and subjects to the natural boundaries and the general initial condition. We obtain explicit analytical expressions in terms of the Fox $\mathbf{H}$ functions for the probability distribution. In addition, we analyze the first passage time and the second movement distribution for the case characterized by the absence of absorbent term and external force for a semiinfinite interval with absorbing boundary condition.
\end{abstract}

Keywords-anomalous diffusion; fractional diffusion; green function; fox function

\section{INTRODUCTION}

Anomalous diffusion is one of the most ubiquitous phenomena in nature [1]. It is present in a wide variety of physical situations. For instance, surface growth, transport of fluid in porous media [2], two-dimensional rotating flow [3], subrecoil laser cooling [4], diffusion on fractals [5], or even in multidisciplinary areas such as econophysics [6-8]. The properties concerning these equations have also been investigated. For instance, in [9] boundary values problems for fractional diffusion equations are studied, in [10] a fractional Fokker-Planck equation is derived from a generalized master equation, in [11] the behavior of fractional diffusion at the origin is analyzed and a connection between the Fox $\mathrm{H}$ functions and the fractional diffusion equations was investigated in [12]. Also a generalization of Brownian motion to multidimensional anomalous diffusion is considered by using fractional differential equation in [13, 14]. Analytical solution of fractional Navier-Stokes equation is investigated by using modified Laplace decomposition method in [15]. In [16] the maximum principles for solutions of the linear fractional diffusion equations are derived, in [17], the regional controllability for the Riemann-Liouville time fractional diffusion systems is analysed, in [18] a harmonic analysis of random fractional diffusion-wave equations is done, in [19] the Cauchy problem for fractional diffusion equations is discussed, and second order accuracy finite difference methods for spacefractional partial differential equations are proposed in [20]. The space-time fractional nonlinear Schrödinger equation is solved by mean of on the fractional Riccati expansion method [21]. Analytical solution of time-fractional Drinfeld-SokolovWilson system is obtained by using residual power series method [22].
In this direction, we dedicate this work to investigate a fractional diffusion equation which employs space and time fractional derivatives by taking a time-dependent diffusion coefficient, an absorbent or sources term and an external force into account. More precisely, we focus our attention on the following equation

$$
\begin{aligned}
\frac{\partial^{\gamma}}{\partial t^{\gamma}} \rho(x, t) & =\int_{0}^{t} d t^{\prime} D\left(t-t^{\prime}\right) \frac{\partial^{\mu}}{\partial x^{\mu}} \rho\left(x, t^{\prime}\right) \\
& -\frac{\partial}{\partial x}[F(x) \rho(x, t)]-\int_{0}^{t} d t^{\prime} a\left(t-t^{\prime}\right) \rho\left(x, t^{\prime}\right)
\end{aligned}
$$

With $0<\gamma, 1,0<\mu, 2$, where $D(t)$ is a time dependent diffusion coefficient, $F(x)$ is an external force, $a(t)$ is a time dependent absorbent term, which may be related to a reaction diffusion process. Here we use the Caputo operator for the fractional derivative with respect to time $t$ and the Riesz-Weyl operator for the fractional derivative with respect to spatial $x$ [23] and we work with the positive spatial variable $x$. Later on, we will extend the results to the entire real $x$-axis by the use of symmetry (in other words, we are working with $\partial^{\mu} / \partial|x|^{\mu}$ ). The presence of the reaction term like the one presents in the above equation may be useful to investigate several situations by choosing an appropriated $a(t)$. For example, catalytic processes in regular, heterogeneous, or disordered systems [24, 25].

The plan of this work is to start by considering (1) without external force and absorbent term. Then we consider (1) in the presence of the absorbent term $a(t)=a t^{\beta-1} / \Gamma(\beta)$ without external force. After that we incorporate the external force $F(x)=-\mathrm{K} x$ in our analysis. In all the above cases, (1) satisfies to the generic initial condition $\rho(x, 0)=\tilde{\rho}(x)(\tilde{\rho}(x)$ is a given function), and the natural boundary condition $\rho( \pm \infty, t)=0$. The remainder of this paper goes as follow. In Sec.2, we obtain the exact solutions for the previous cases. In Sec.3, we present our conclusions.

\section{SOLUTION FOR THE FRACTIONAL DIFFUSION EQUATION}

Let us start our analysis by considering (1) in the absence of the external force and the absorbent term. Thus (1) reads 


$$
\frac{\partial^{\gamma}}{\partial t^{\gamma}} \rho(x, t)=\int_{0}^{t} d t^{\prime} D\left(t-t^{\prime}\right) \frac{\partial^{\mu}}{\partial x^{\mu}} \rho\left(x, t^{\prime}\right) .
$$

Notice that for $\gamma=1, \mu=2$, (2) reduces to the usual diffusion equation taking memory effect into account, which can be obtained from a dichotomous random process [26]. By applying the Laplace and Fourier transforms, and employing Riesz representation for the spatial fractional derivatives, we may simplify (2), which is an integral order differential equation, to the following algebraic equation

$$
s^{\gamma} \hat{\tilde{\rho}}(k, s)-s^{\gamma-1} \hat{\rho}(k, 0)=-\tilde{D}(s)|k|^{\mu} \hat{\tilde{\rho}}(k, s)
$$

where

$$
\tilde{\rho}(x, s)=\mathrm{L}\{\rho(x, t)\}=\int_{0}^{\infty} \rho(x, t) e^{-s t} d t
$$$$
\tilde{D}(s)=\mathrm{L}\{D(t)\}=\int_{0}^{\infty} D(t) e^{-s t} d t=D s^{-\alpha}
$$

$\hat{\rho}(k, t)=\mathrm{F}\{\rho(x, t)\}=\int_{-\infty}^{+\infty} \rho(x, t) e^{-i k x} d x$. Then, (3) can be solved by the Green function method [27]. So we have

$$
\rho(x, t)=\int_{-\infty}^{+\infty} d x^{\prime} \mathrm{G}\left(x-x^{\prime}, t\right) \rho\left(x^{\prime}, 0\right) .
$$

Applying the Fourier and Laplace transform in (4), we obtain the solution which is given by

$$
\begin{gathered}
\hat{\tilde{\rho}}(k, s)=\hat{\tilde{\mathrm{G}}}(k, s) \hat{\rho}(k, 0), \\
\hat{\widetilde{\mathrm{G}}}(k, s)=\frac{s^{\gamma-1}}{s^{\gamma}+\tilde{D}(s)|k|^{\mu}},
\end{gathered}
$$

where $\hat{\rho}(k, 0)$ is the Fourier transform of the initial condition and $\hat{\mathrm{G}}(k, t)$ is the Green function of (2) in the Fourier-Laplace space. Applying the inverse of Laplace transform, we obtain

$$
\widehat{\mathrm{G}}(k, t)=E_{\gamma+\alpha, 1}\left(-D|k|^{\mu} t^{\gamma+\alpha}\right)
$$

where $E_{\alpha, \beta}(x)$ is the Mittag-Leffler function. In order to perform the inverse of Fourier transform, we express $\hat{\widetilde{G}}(k, t)$ in the terms of Fox function, i.e. $H_{p}^{m} \quad{ }_{q}^{n}\left[\left.x\right|_{\left(b_{(1)}, B(1)\right), \ldots,\left(b_{(q)}, B(q)\right)} ^{\left(a_{(1)}, A(1)\right), \ldots,\left(a_{(p)}, A(p)\right)}\right]$ [28]. So (7) can be written as follows

$$
\widehat{\mathrm{G}}(k, t)=H_{1}^{1} \quad{ }_{2}^{1}\left[\left.D|k|^{\mu} t^{\gamma+\alpha}\right|_{(0,1),(0, \gamma+\alpha)} ^{(0,1)}\right] .
$$

This solution recovers the usual one for $(\mu, \gamma)=(2,1)$ and for $\mu \neq 2$ it extends the results found in [29]. Note that the Mellin transform of the Fourier transform of $f(x)$ is an even function

$$
\begin{aligned}
\mathrm{M}\{\mathrm{F}[f(x)](k)\}(z) & =2 \sqrt{\frac{\pi}{2}} \mathrm{M}\left\{\mathrm{F}_{c}[f(x)](k)\right\}(z) \\
& =2 \Gamma(z) \cos \left(\frac{z}{2} \pi\right) \mathrm{M}\{f(x)\}(1-z) .
\end{aligned}
$$

We first evaluate the Mellin transform of (8) to find the Mellin transform of $\mathrm{G}(x, t)$. The Mellin transform then only need to be inverted to find Fourier inverse, $\mathrm{G}(x, t)$. To find the Mellin transform of (8), we note the Mellin transform of a Fox function is given by [30]

$\mathrm{M}\left\{H_{p}^{m} \quad q_{q}^{n}\left[\left.\operatorname{ax}\right|_{\left(b_{q}, \beta_{q}\right)} ^{\left(a_{p}, \alpha_{p}\right)}\right]\right\}_{(z)}=a^{-z} \frac{\prod_{j=1}^{m} \Gamma\left(b_{j}+\beta_{j} z\right) \Pi_{j=1}^{n} \Gamma\left(1-a_{j}-\alpha_{j} z\right)}{\prod_{j=m+1}^{q} \Gamma\left(1-b_{j}-\beta_{j} z\right) \Pi_{j=n+1}^{p} \Gamma\left(a_{j}+\alpha_{j} z\right)}$,

when the following conditions are met

$$
\delta=\sum_{j=1}^{q} \beta_{j}-\sum_{j=1}^{m} \alpha_{j}>0
$$

$$
A=\sum_{j=1}^{n} \alpha_{j}-\sum_{j=n+1}^{p} \alpha_{j}+\sum_{j=1}^{m} \beta_{j}-\sum_{j=m+1}^{q} \beta_{j}>0
$$

$$
|\arg (a)|<\frac{1}{2} A \pi
$$

$$
-\min _{1 \leq j \leq m}\left[\mathrm{R}\left(\frac{b_{j}}{\beta_{j}}\right)\right]<\mathrm{R}(z)<\min _{1 \leq j \leq n}\left[\mathrm{R}\left(\frac{1-a_{j}}{\alpha_{j}}\right)\right] .
$$

By applying this procedure, we obtain (see Figure I)

$$
\mathrm{G}(x, t)=\frac{1}{2 \mu \sqrt{\pi}\left(D t^{\gamma+\alpha}\right)^{1 / \mu}} H_{2}^{2}{ }_{3}^{1}\left[\left.\frac{|x|}{2\left(D t^{\gamma+\alpha}\right)^{1 / \mu}}\right|_{\left(0, \frac{1}{2}\right)\left(1-\frac{1}{\mu}, \frac{1}{\mu}\right)\left(\frac{1}{2}, \frac{1}{2}\right)} ^{\left(1-\frac{1}{\mu}, \frac{1}{\mu}\right),\left(1-\frac{\gamma+\alpha}{\mu}\right)}\right]
$$

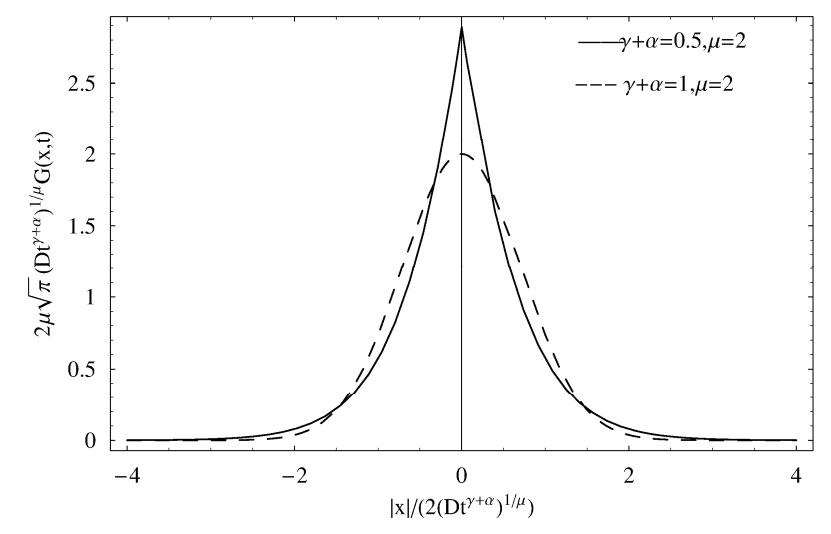

FIGURE I. THE BEHAVIOR OF GREEN FUNCTION G( $x, t)$ IN (15) IS ILLUSTRATED BY CONSIDERING $2 \mu \sqrt{\pi}\left(D t^{\gamma+\alpha}\right)^{1 / \mu} \mathrm{G}(x, t)$ VERSUS $\frac{|x|}{2\left(D t^{\gamma+\alpha}\right)^{1 / \mu}}$ FOR TYPICAL VALUES OF $\gamma+\alpha$ AND $\mu$ 
In Figure I, we show the behavior of the above equation for typical values of $\mu, \gamma$ and $\alpha$. Note that the Green function obtained here leads to an anomalous spreading of the initial condition due to the presence of the spatial and time fractional derivatives and a memory effect. This feature can be verified, for simplicity, by analyzing the second movement of (15) for particular case $\mu=2$. For this case, it is given by

$$
<x^{2}>=\frac{2}{\Gamma(\gamma+1)} \int_{0}^{t} d t^{\prime}\left(t-t^{\prime}\right)^{\gamma} D\left(t^{\prime}\right)
$$

For the initial condition $\rho(x, 0)=\delta(x)$ and the diffusion coefficient $D(t)=D t^{\alpha-1} / \Gamma(\alpha)$. Figure II shows the behavior of $\left\langle x^{2}\right\rangle$ versus $t$, which illustrates how (16) evolves on the time by considering, for simplicity, $D=1$ for typical values of $\gamma$ and $\alpha$.

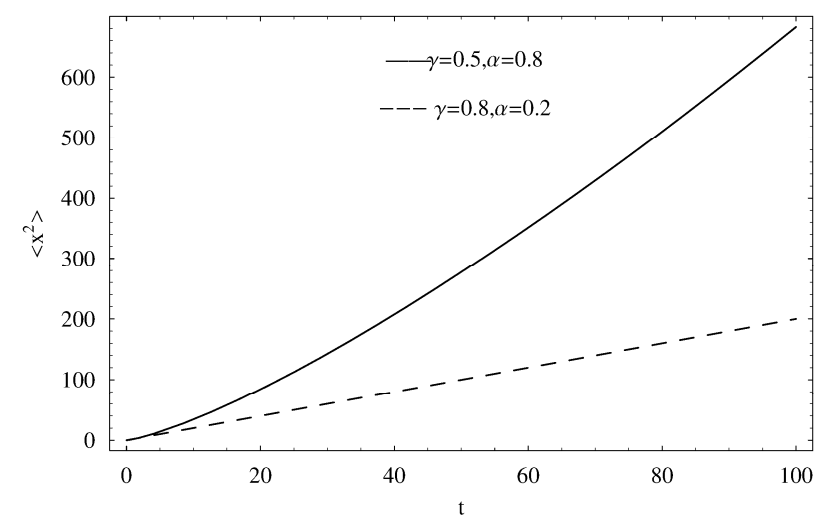

FIGURE II. THE BEHAVIOR OF $<\mathrm{X}^{2}>$ VERSUS T

In Figure II, we can see for small time $<x^{2}>$ is dominated by the initial distance, and for large time the rate of $\left\langle x^{2}\right\rangle$ is less than that of the intermediate time. This behaviors are verified in turbulent processes [31]. At this point, by submitting (15) and the initial condition $\rho(x, 0)=\tilde{\rho}(x)$ into (4), we can get the solution for (2), which is given by

$$
\begin{aligned}
& \rho(x, t)= \\
& \int_{-\infty}^{+\infty} d x^{\prime} \frac{1}{2 \mu \sqrt{\pi}\left(D t^{\gamma+\alpha}\right)^{1 / \mu}} H_{2}^{2}{ }_{3}^{1}\left[\left.\frac{\left|x-x^{\prime}\right|}{2\left(D t^{\gamma+\alpha}\right)^{1 / \mu}}\right|_{\left(0, \frac{1}{2}\right)\left(1-\frac{1}{\mu}, \frac{1}{\mu}\right)\left(\frac{1}{2}, \frac{1}{2}, \frac{1}{2}\right),\left(1-\frac{\gamma+\alpha}{\mu}\right)} ^{(1)}\right] \tilde{\rho}\left(x^{\prime}\right) .
\end{aligned}
$$

The result obtained here can be rated to several results presented in $[10,12,32]$.

In this direction, by using the previous result and the method of images [27], we may find the solution when the boundary condition is defined in a semi-infinity interval, i.e. $\rho(0, t)=\rho(\infty, t)=0$. In particular, the solution taking this boundary condition into account, in the absence of the absorbent term and external force, is given by

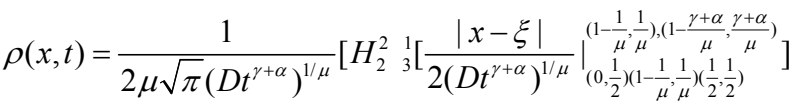

$$
\begin{aligned}
& -H_{2}^{2}{ }_{3}^{1}\left[\left.\frac{|x+\xi|}{2\left(D t^{\gamma+\alpha}\right)^{1 / \mu}}\right|_{\left(0, \frac{1}{2}\right)\left(1-\frac{1}{\mu}, \frac{1}{\mu}, \frac{1}{\mu}, \frac{1}{2}, \frac{1}{2}\right)} ^{\left(1-\frac{1}{\mu}, \frac{1}{\mu}\right),\left(1, \frac{\gamma+\alpha}{\gamma}\right)}\right]
\end{aligned}
$$

For the initial condition $\rho(x, 0)=\delta(x-\xi)$. This result extends results found in $[33,34]$ and the first passage time distribution for the system governed by this case, using the definition $\mathrm{F}(t)=-\int_{0}^{\infty} \frac{\partial \rho(x, t)}{\partial t} d x$ employed in [35], is given by

$\mathrm{F}(t)=\frac{(\gamma+\alpha) \xi}{\mu^{2} \sqrt{\pi} t\left(D t^{\gamma+\alpha}\right)^{1 / \mu}} H_{2}^{2}{ }_{3}^{1}\left[\left.\frac{\xi}{2\left(D t^{\gamma+\alpha}\right)^{1 / \mu}}\right|_{\left(0, \frac{1}{2}\right)\left(1-\frac{1}{\mu}, \frac{1}{\mu}, \frac{1}{\mu}\right)\left(\frac{1}{2}, \frac{1}{2}\right)} ^{\left(1-\frac{1}{\mu}\right),\left(1-\frac{\gamma+\alpha}{\gamma}\right)}\right]$,

Which has the asymptotic behavior $\mathrm{F}(t) \sim 1 / t^{1+(\gamma+\alpha) / \mu}$ for large time. In Figure III, the behavior of the first passage time distribution $\mathrm{F}(t)$ in (19) is illustrated by considering $\left[\mu^{2} \sqrt{\pi} t /(2(\gamma+\alpha))\right] \mathrm{F}(t)$ versus $\xi /\left(2\left(D t^{\gamma+\alpha}\right)^{1 / \mu}\right)$, for typical values of $\gamma+\alpha$ and $\mu$.

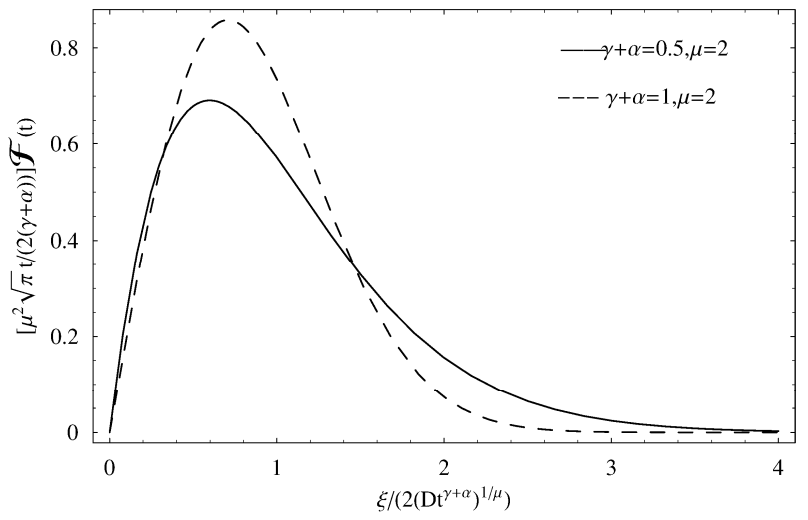

FIGURE III. THE BEHAVIOR OF THE FIRST PASSAGE TIME DISTRIBUTION F $(t)$ IN (19)

Let us go back to (1) and consider $a(t)=a t^{\beta-1} / \Gamma(\beta)$. Then, (1) reads

$\frac{\partial^{\gamma}}{\partial t^{\gamma}} \rho(x, t)=\int_{0}^{t} d t^{\prime} D\left(t-t^{\prime}\right) \frac{\partial^{\mu}}{\partial x^{\mu}} \rho\left(x, t^{\prime}\right)-\frac{a}{\Gamma(\beta)} \int_{0}^{t} d t^{\prime}\left(t-t^{\prime}\right)^{\beta-1} \rho\left(x, t^{\prime}\right)$.

By using the Laplace and Fourier transforms, at the same time employing Riesz representation for the spatial fractional derivatives, we have

$$
s^{\gamma} \hat{\tilde{\rho}}(k, s)-s^{\gamma-1} \hat{\rho}(k, 0)=-\tilde{D}(s)|k|^{\mu} \hat{\tilde{\rho}}(k, s)-\tilde{a}(s) \hat{\tilde{\rho}}(k, s),
$$


where $\tilde{D}(s)=D s^{-\alpha}, \tilde{a}(s)=a s^{-\beta}$ and $\hat{\rho}(k, 0)$ is the Fourier transform of the initial condition. The solution of this equation, for simplicity, by considering $\hat{\rho}(k, 0)=1$, is given by

$$
\hat{\tilde{\rho}}(k, s)=\frac{s^{\gamma-1}}{s^{\gamma}+\tilde{D}(s)|k|^{\mu}+\tilde{a}(s)} .
$$

Then, we employ the procedure presented in [36], where an explanation of how to get the series expansion in terms of Fox $\mathrm{H}$ function can be found. By applying this procedure, we obtain

$$
\hat{\tilde{\rho}}(k, s)=\sum_{j=0}^{\infty} \frac{(-a)^{j} s^{\gamma+\alpha+(\alpha-\beta) j-1}}{\left(s^{\gamma+\alpha}+D|k|^{\mu}\right)^{j+1}} .
$$

Applying the inverse of Laplace transform on the above equation, we can obtain

$$
\begin{aligned}
\hat{\rho}(k, t) & =\sum_{j=0}^{\infty} \frac{\left(-a t^{\beta+\gamma}\right)^{j}}{j !} E_{\gamma+\alpha,(\beta-\alpha) j+1}^{(j)}\left(-D|k|^{\mu} t^{\gamma+\alpha}\right) \\
& =\sum_{j=0}^{\infty} \frac{\left(-a t^{\beta+\gamma}\right)^{j}}{j !} H_{1}^{1}{ }_{2}^{1}\left[\left.D|k|^{\mu} t^{\gamma+\alpha}\right|_{(0,1)(-(\beta+\gamma) j, \gamma+\alpha)} ^{(-j, 1)}\right] .
\end{aligned}
$$

Then, following the same procedure as in the first case to find the Fourier inverse of $\hat{\rho}(k, t)$, we have

$$
\begin{aligned}
& \rho(x, t)= \\
& \sum_{j=0}^{\infty} \frac{\left(-a t^{\beta+\gamma}\right)^{j}}{2 \mu j ! \sqrt{\pi}\left(D t^{\gamma+\alpha}\right)^{1 / \mu}} H_{2}^{2}{ }_{3}^{1}\left[\left.\frac{|x|}{2\left(D t^{\gamma+\alpha}\right)^{1 / \mu}}\right|_{\left(0, \frac{1}{2}\right)\left(1+j-\frac{1}{\mu}, \frac{1}{\mu}\right)\left(\frac{1}{2}, \frac{1}{2}\right)} ^{\left.\left(1-\frac{1}{\mu}, \frac{1}{\mu}\right),\left(1+(\beta+\gamma),-\frac{\gamma+\alpha}{\mu}, \frac{\gamma+\alpha}{\mu}\right)\right] .}\right.
\end{aligned}
$$

Note that the result got here for a bi-fractional reaction diffusion equation recovers the solution for the usual one.

Let us incorporate the external force $F(x)=-\mathrm{K} x$ into the previous calculations. For this case, (1) reads

$$
\begin{aligned}
& \frac{\partial^{\gamma}}{\partial t^{\gamma}} \rho(x, t)= \\
& \int_{0}^{t} d t^{\prime} D\left(t-t^{\prime}\right) \frac{\partial^{\mu}}{\partial x^{\mu}} \rho\left(x, t^{\prime}\right)+\mathrm{K} \frac{\partial}{\partial x}[x \rho(x, t)]-\frac{a}{\Gamma(\beta)} \int_{0}^{t} d t^{\prime}\left(t-t^{\prime}\right)^{\beta-1} \rho\left(x, t^{\prime}\right) .
\end{aligned}
$$

Following the procedure employed above to the case without external forces, we also use the Fourier and Laplace transforms to simplify our study. By using these integral transforms, (26) can be simplified to

$$
\hat{\tilde{\rho}}(k, s)=\sum_{n=0}^{\infty} \frac{1}{n !}\left(\frac{\tilde{D}(s)|k|^{\mu}}{\mathrm{K} \mu}\right)^{n} e^{-\frac{\left.\tilde{D}(s) k\right|^{\mu}}{\mathrm{K} \mu}} \frac{s^{\gamma-1}}{s^{\gamma}+n \mu \mathrm{K}+\tilde{a}(s)} .
$$

By applying the inverse of Laplace transform on (27) as above, we can obtain

$$
\begin{aligned}
\hat{\rho}(k, t)= & \sum_{n=0}^{\infty} \sum_{j=0}^{\infty} \int_{0}^{t} d t^{\prime} \frac{\left[-a\left(t-t^{\prime}\right)^{\beta+\gamma}\right]^{j}}{n ! j ! t^{\prime}} H_{1}^{1}{ }_{1}^{0}\left[\left.\frac{\mathrm{D}|k|^{\mu}}{\mathrm{K} \mu} t^{\prime \alpha}\right|_{(n, 1)} ^{(0,-\alpha)}\right] \\
& \times E_{\gamma, 1+\beta j}^{(j)}\left(-n \mu \mathrm{K}\left(t-t^{\prime}\right)^{\gamma}\right) .
\end{aligned}
$$

Here, we used the property of the Laplace transform of convolution formula, i.e. $\mathrm{L}[f * g]=f \cdot g$, where

$$
f * g=\int_{0}^{t} d t^{\prime} f\left(t-t^{\prime}\right) g\left(t^{\prime}\right)
$$

In order to get the solution, we need to get the inverse of Fourier transform of $\hat{\mathrm{G}}(k, t)$. Therefore, we only need to perform the inverse of Fourier transform on $H_{1}^{1}{ }_{1}^{0}\left[\left.\frac{D|k|^{\mu} t^{\prime \alpha}}{\mathrm{K} \mu}\right|_{(n, 1)} ^{(0,-\alpha)}\right]$. Following the same procedure employed in the first case, we have

$$
\mathrm{F}^{-1}\left\{H_{1}^{1}{ }_{1}^{0}\left[\left.\frac{D|k|^{\mu} t^{\prime \alpha}}{\mathrm{K} \mu}\right|_{(n, 1)} ^{0,-\alpha}\right]\right\}=\frac{1}{\mu \sqrt{\pi}|x|} H_{2}^{1}{ }_{2}^{1}\left[\left.\frac{|x|}{2}\left(\frac{\mathrm{K} \mu}{D t^{\prime \alpha}}\right)^{1 / \mu}\right|_{\left(\frac{1}{2}, \frac{1}{2}\right)\left(1, \frac{1}{2}\right)} ^{\left.(1-n), \frac{1}{\mu}\right)\left(0, \frac{\alpha}{\mu}\right)}\right]
$$

and

$$
\begin{aligned}
\rho(x, t)= & \sum_{n=0}^{\infty} \sum_{j=0}^{\infty} \int_{0}^{t} d t^{\prime} \frac{\left(-a\left(t-t^{\prime}\right)^{\beta+\gamma}\right)^{j}}{\mu n ! j ! \sqrt{\pi}|x| t^{\prime}} H_{2}^{1}{ }_{2}^{1}\left[\left.\frac{|x|}{2}\left(\frac{\mathrm{K} \mu}{D t^{\prime \alpha}}\right)^{1 / \mu}\right|_{\left(\frac{1}{2}, \frac{1}{2}\right)\left(1, \frac{1}{2}\right)} ^{\left(1-n, \frac{1}{\mu}\right)\left(0, \frac{\alpha}{\mu}\right)}\right] \\
& \times E_{\gamma, 1+\beta j}^{(j)}\left(-n \mu \mathrm{K}\left(t-t^{\prime}\right)^{\gamma}\right) .
\end{aligned}
$$

The above equation in the absence of absorbent term recovers the results found in [37], i.e. $a(t)=0$. Note that the solution of (26) in the absence of absorbent term is a stationary one given in terms of the Lévy distribution. This feature is a characteristic of the presence of the spatial derivatives in the diffusion equation which changes the probability for a jump length (see [38] and references therein).

\section{SUMMARY AND CONCLUSIONS}

We have worked out a generalized diffusion equation which presents space and time fractional derivatives and takes an absorbent term and the external force into account. We have first analyzed the case characterized by the absence of external forces and the absorbent term. For this case, we have obtained the exact solution and expressed it in terms of a Fox function. Furthermore, we have considered the second moment for this case and obtained the first passage time distribution by taking the boundary conditions $\rho(0, t)=\rho(\infty, t)=0$ into account. Then we consider (2) with an absorbent term $a(t)=a t^{\beta-1} / \Gamma(\beta)$. Subsequently, we have incorporated the external force $F(x)=-\mathrm{K} x$ to the previous situations in which the absorbent 
term is present. For the third case, we have also discussed the stationary solution which emerges from $\alpha(t)=0$. In this sense, the present results may be considered as an extension to a broad context of the analysis for the time fractional diffusion equations. Finally, we expect that the results obtained here may be useful to the discussion of the anomalous diffusion systems where fractional diffusion equations play an important role.

\section{ACKNOWLEDGMENT}

We would like to thank the Natural Science Foundation of Zhejiang (Grant No. LY17A010020) for partial financial support and be grateful to the anonymous referees for useful comments and suggestions.

\section{REFERENCES}

[1] J. Bouchaud and A. Georges, "Anomalous diffusion in disordered media: statistical mechanisms, models and physical applications," Physics reports, vol. 195, pp. 127-293, 1990.

[2] H. Spohn, "Surface dynamics below the roughening transition," Journal de Physique I, vol. 3, pp. 69-81, 1993.

[3] T. H. Solomon, E. R. Weeks and H. L. Swinney, "Observation of anomalous diffusion and Lévy flights in a two-dimensional rotating flow," Phys Rev Lett, vol. 71, p. 3975, 1993.

[4] F. Bardou, J. P. Bouchaud, O. Emile, A. Aspect, and C. CohenTannoudji, "Subrecoil laser cooling and Lévy flights," Phys Rev Lett, vol. 72 , p. 203, 1994

[5] J. Stephenson, "Some non-linear diffusion equations and fractal diffusion," Physica A: Statistical Mechanics and its Applications, vol. 222, pp. 234-247, 1995.

[6] V. Plerou, P. Gopikrishnan, L. A. N. Amaral, X. Gabaix, and H. E. Stanley, "Economic fluctuations and anomalous diffusion," Phys Rev E, vol. 62, p. R3023, 2000.

[7] L. Longjin, F. Ren and W. Qiu, "The application of fractional derivatives in stochastic models driven by fractional Brownian motion," Physica A: Statistical Mechanics and its Applications, vol. 389, pp. 4809-4818, 2010.

[8] L. Lv, J. Xiao, L. Fan, and F. Ren, "Correlated continuous time random walk and option pricing," Physica A: Statistical Mechanics and its Applications, vol. 447, pp. 100-107, 2016.

[9] R. Metzler and J. Klafter, "The random walk's guide to anomalous diffusion: a fractional dynamics approach," Physics reports, vol. 339, pp. 1-77, 2000.

[10] R. Metzler and T. F. Nonnenmacher, "Space-and time-fractional diffusion and wave equations, fractional Fokker-Planck equations, and physical motivation," Chem Phys, vol. 284, pp. 67-90, 2002.

[11] B. West, M. Bologna and P. Grigolini, Physics of fractal operators: Springer Science \& Business Media, 2012.

[12] F. Mainardi, G. Pagnini and R. K. Saxena, "Fox H functions in fractional diffusion," J Comput Appl Math, vol. 178, pp. 321-331, 2005.

[13] V. V. Uchaikin, "Multidimensional symmetric anomalous diffusion," Chem Phys, vol. 284, pp. 507-520, 2002.

[14] L. Lv, F. Ren, J. Wang, and J. Xiao, "Correlated continuous time random walk with time averaged waiting time," Physica A: Statistical Mechanics and its Applications, vol. 422, pp. 101-106, 2015.

[15] S. Kumar, D. Kumar, S. Abbasbandy, and M. M. Rashidi, "Analytical solution of fractional Navier-Stokes equation by using modified Laplace decomposition method," Ain Shams Engineering Journal, vol. 5, pp. 569-574, 2014.

[16] M. Al-Refai and Y. Luchko, "Maximum principle for the fractional diffusion equations with the Riemann-Liouville fractional derivative and its applications," Fract Calc Appl Anal, vol. 17, pp. 483-498, 2014.

[17] F. Ge, Y. Chen and C. Kou, "Regional controllability analysis of fractional diffusion equations with Riemann-Liouville time fractional derivatives," Automatica, vol. 76, pp. 193-199, 2017.
[18] V. V. Anh and N. N. Leonenko, "Harmonic analysis of random fractional diffusion--wave equations," Appl Math Comput, vol. 141, pp. $77-85,2003$.

[19] S. D. Eidelman and A. N. Kochubei, "Cauchy problem for fractional diffusion equations," J Differ Equations, vol. 199, pp. 211-255, 2004.

[20] Y. Takeuchi, Y. Yoshimoto and R. Suda, "Second order accuracy finite difference methods for space-fractional partial differential equations," J Comput Appl Math, vol. 320, pp. 101-119, 2017.

[21] E. A. Abdel-Salam, E. A. Yousif and M. A. El-Aasser, "Analytical Solution of the Space-Time Fractional Nonlinear Schrödinger Equation," Rep Math Phys, vol. 77, pp. 19-34, 2016.

[22] H. M. Jaradat, S. Al-Shara, Q. J. Khan, M. Alquran, and K. Al-Khaled, "Analytical solution of time-fractional Drinfeld-Sokolov-Wilson system using residual power series method," IAENG International Journal of Applied Mathematics, vol. 46, pp. 64-70, 2016.

[23] I. Podlubny, Fractional differential equations vol. 198: Academic press, 1998.

[24] P. A. Alemany, D. H. Zanette and H. S. Wio, "Time-dependent reactivity for diffusion-controlled annihilation and coagulation in two dimensions," Phys Rev E, vol. 50, p. 3646, 1994.

[25] B. J. West, P. Grigolini, R. Metzler, and T. F. Nonnenmacher, "Fractional diffusion and Lévy stable processes," Phys Rev E, vol. 55, p. 99, 1997.

[26] P. Allegrini, P. Grigolini and B. J. West, "Dynamical approach to Lévy processes," Phys Rev E, vol. 54, p. 4760, 1996.

[27] H. Feshbach and P. M. Morse, Methods of theoretical physics: McGrawHill Interamericana, 1953.

[28] A. M. Mathai and R. K. Saxena, "The H function with applications in statistics and other disciplines," 1978.

[29] R. Metzler, E. Barkai and J. Klafter, "Anomalous diffusion and relaxation close to thermal equilibrium: a fractional Fokker-Planck equation approach," Phys Rev Lett, vol. 82, p. 3563, 1999.

[30] H. M. Srivastava, K. C. Gupta and S. P. Goyal, The H-functions of one and two variables, with applications: South Asian Publishers, 1982.

[31] G. Boffetta and I. M. Sokolov, "Relative dispersion in fully developed turbulence: the Richardson's law and intermittency corrections," Phys Rev Lett, vol. 88, p. 094501, 2002.

[32] [32] F. Mainardi, Y. Luchko and G. Pagnini, "The fundamental solution of the space-time fractional diffusion equation," Fract Calc Appl Anal, vol. 4, pp. 153-192, 2001.

[33] G. Rangarajan and M. Ding, "Anomalous diffusion and the first passage time problem," Phys Rev E, vol. 62, p. 120, 2000.

[34] L. Lv, J. Xiao, L. Zhang, and L. Gao, "Solutions for a generalized fractional anomalous diffusion equation," J Comput Appl Math, vol. 225 pp. 301-308, 2009.

[35] H. Risken, Fokker-Planck Equation: Springer, 1996.

[36] T. Langlands, "Solution of a modified fractional diffusion equation," Physica A: Statistical Mechanics and its Applications, vol. 367, pp. 136144, 2006.

[37] R. Metzler, E. Barkai and J. Klafter, "Anomalous transport in disordered systems under the influence of external fields," Physica A: Statistical Mechanics and its Applications, vol. 266, pp. 343-350, 1999.

[38] A. Schot, M. K. Lenzi, L. R. Evangelista, L. C. Malacarne, R. S. Mendes, and E. K. Lenzi, "Fractional diffusion equation with an absorbent term and a linear external force: Exact solution," Phys Lett A, vol. 366, pp. 346-350, 2007. 